\title{
Case Report: Visceral Leishmaniasis Falsely Diagnosed as Q Fever
}

\author{
Ankush Dhariwal, ${ }^{1 *}$ Sarah Jawad, ${ }^{2}$ and Tihana Bicanic ${ }^{2}$ \\ ${ }^{1}$ Barts Health NHS Trust, London, United Kingdom; ${ }^{2}$ St George's University Hospitals NHS Foundation Trust, London, United Kingdom
}

\begin{abstract}
Visceral leishmaniasis (VL) is a systemic infection caused by the protozoal parasite Leishmania, spread via the bloodstream to the reticuloendothelial system, through the bite of the sand fly. It is endemic in parts of Africa, South America, Asia, and Europe, including the Mediterranean. Here, we describe a case of VL that was initially diagnosed as $Q$ fever based on positive Coxiella burnetii serology and showed a partial response to doxycycline treatment.
\end{abstract}

\section{CASE PRESENTATION}

A 21-year-old man presented to a specialist infectious diseases service at a London hospital with fever, weight loss, and night sweats of 2-week duration, accompanied by abdominal discomfort. He was born in the United Kingdom and had lived his life between the United Kingdom and Spain, predominantly Barcelona and Valencia. His only other travel was a holiday to Morocco 2 years before presentation. He worked in a pastry factory and had a healthy pet dog. He had never worked with animals or consumed unpasteurized dairy or raw meat. On systems review, he denied rashes, arthralgia, or respiratory symptoms.

His medical history was notable for a similar presentation 6 months ago in Spain, with fever, pancytopenia, and splenomegaly. Among numerous investigations, Coxiella burnetii serology (immunofluorescence assay [IFA], Vircell) was positive (phase $1 \operatorname{lgG} 1: 180$; phase $2 \lg$ G 1:640), and he was treated for $Q$ fever with 2 weeks of doxycycline and his fever resolved completely.

On examination, he was febrile at $38.7^{\circ} \mathrm{C}$ and looked generally unwell. He had no rashes, and cardiorespiratory examination was unremarkable. His liver was palpable at $3 \mathrm{~cm}$ below the costal margin, and he had splenomegaly extending across the midline, with palpable subcentimeter cervical and inguinal lymph nodes.

Baseline investigations demonstrated pancytopenia (hemoglobin $86 \mathrm{~g} / \mathrm{L}$, platelets $88 \times 10^{6} / \mathrm{L}$, white cell count $0.9 \times$ $10^{6} / \mathrm{L}$, and neutrophils $\left.0.3 \times 10^{6} / \mathrm{L}\right)$. Liver and renal function tests were normal, and C-reactive protein was raised at 137 $\mathrm{mg} / \mathrm{L}$. A blood film showed rouleaux formation in keeping with an inflammatory state, and no abnormal cells were seen. Thick and thin films for malaria, HIV serology, and four sets of blood cultures were all negative.

Computed tomography of his abdomen and pelvis demonstrated hepatomegaly and massive splenomegaly of $28 \mathrm{~cm}$, with no other abnormal findings (Figure 1). He continued to spike temperatures over $38^{\circ} \mathrm{C}$, with no response to piperacillin-tazobactam (given as empirical treatment of neutropenic fever).

In the context of pancytopenia, hepatosplenomegaly, and B symptoms (fever, weight loss, and night sweats), the differential diagnosis included infection or hematological malignancy. Two bone marrow aspirates from day 10 and day 11 of

*Address correspondence to Ankush Dhariwal, St Bartholomew's Hospital, W Smithfield, London EC1A 7BE, United Kingdom. E-mail: ankush.dhariwal@nhs.net admission demonstrated only reactive changes, and no parasites were seen.

Serology for Bartonella, Brucella, and Rickettsiae was all negative. $Q$ fever serology showed negative $C$. burnetii phase $1 \mathrm{lgG}$ and positive phase 2 lgG (titer 1:160).

Leishmania IFA sent on day 12 with result obtained on day 17 of admission was strongly positive at 1:256. Bone marrow PCR sent on day 11 and reported on day 33 was positive for Leishmania donovani complex DNA, confirming a diagnosis of visceral leishmaniasis (VL). He was commenced on liposomal amphotericin B (AmBisome, Gilead Sciences International Ltd., Cambridge, UK) at $3 \mathrm{mg} / \mathrm{kg}$, given on days $1-5$, day 14 , and day 21 . His fever resolved after 48 hours of treatment, and he was discharged to complete his treatment as an outpatient. He remained well on follow-up at 6 months.

\section{DISCUSSION}

Our case demonstrates two aspects of interest-the potential pitfalls in diagnosing VL and the marked but temporary clinical response our patient demonstrated following initial doxycycline treatment.

Our patient's initial positive $C$. burnetii serology results were more likely to be false positives, for the following reasons, although it is not possible to definitively exclude the possibility of $Q$ fever and VL coinfection in retrospect. His clinical syndrome on first presentation was not in keeping with $Q$ fever. Acute $Q$ fever typically presents with acute pneumonia and hepatitis (although he did have hepatomegaly, he did not have a transaminitis) and does not present with massive splenomegaly or pancytopenia. His repeat serology was not in keeping with chronic $Q$ fever, as phase 1 C. burnetii antibodies are a key feature of chronic $Q$ fever, which were not detected subsequently. On detailed questioning, he had no epidemiological risk factors for acquiring this zoonotic disease, including exposure to farm animals, work in an abattoir, or residence in/travel to a $Q$ fever-endemic area. Finally, his symptoms on both presentations could all be explained by VL.

False-positive $C$. burnetii serology due to other bacterial infections, including Legionella and Bartonella, has been described. $^{1,2}$ To our knowledge, false-positive $C$. burnetii serology due to VL has not been reported, although falsepositive serology for other infections in patients with VL has been described, including leptospirosis ${ }^{3}$ and trypanosomiasis. ${ }^{4}$ Clinicians ought to be aware of this possibility, as patients with unexplained fevers may be investigated with serological tests for atypical bacterial infections, and a positive result may not represent a true positive, especially when the exposure history and clinical syndrome are inconsistent. 


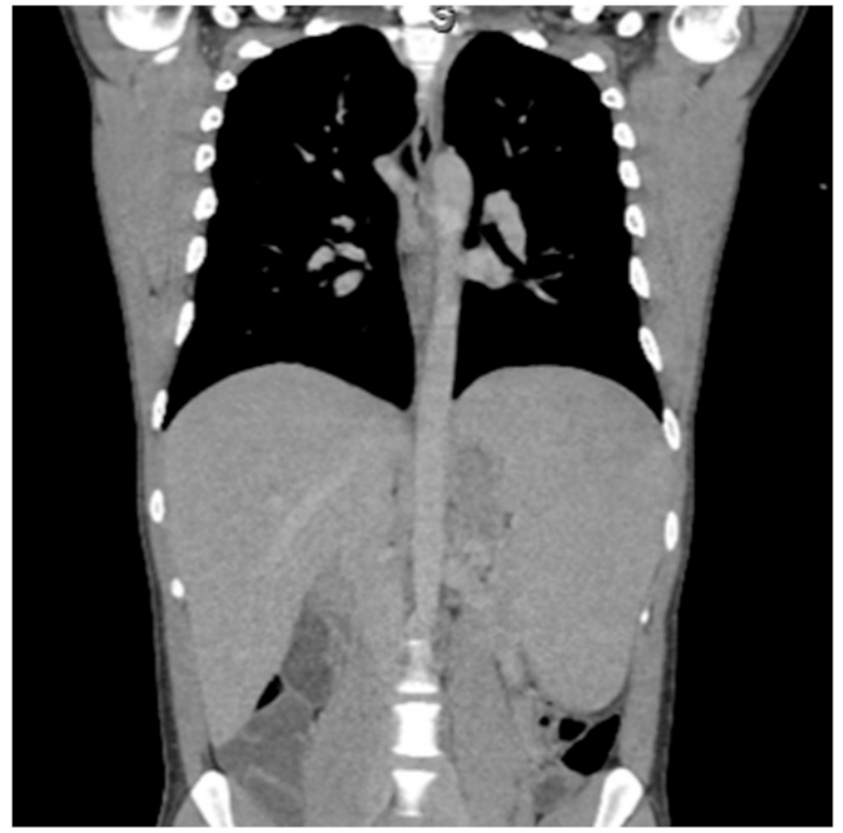

FIGURE 1. Coronal computed tomography scan of chest and abdomen, demonstrating hepatomegaly and massive splenomegaly.

This also highlights the importance of appropriate historytaking before sending specific investigations. Our patient's pancytopenia, fever, and splenomegaly were all in keeping with VL. Symptoms occur because of infection with Leishmania parasites which enter the host when an infected female phlebotomine sand fly takes a blood meal and subsequently infect mononuclear phagocytic cells within the reticuloendothelial system. Leishmania infantum is known to be hypoendemic in Spain ( 0.41 cases per 100,000 people $)^{5}$ and is one of three species within the $L$. donovani complex, the others being $L$. donovani and Leishmania chagasi. $^{6}$

The diagnosis of our patient was delayed by a week as no parasites were seen on two bone marrow aspirates. This serves as a reminder that bone marrow aspirate morphology is not $100 \%$ sensitive for the diagnosis. Reported sensitivity is variable and time dependent, ranging from $55 \%$ to $90 \%,{ }^{7,8}$ and rising proportionally with time spent looking at the slide. ${ }^{9}$ Thus, when the clinical syndrome and epidemiology is in keeping with VL, negative bone marrow aspirate morphology should not be used to exclude the diagnosis, and bone marrow aspirate PCR should be considered.

Our patient described clear clinical improvement in VL symptoms and signs, sustained for a 6-month period, following 2-week doxycycline treatment given for suspected $Q$ fever. The sustained response to doxycycline suggests direct antiparasitic activity against Leishmania, rather than an antiinflammatory or immunomodulatory effect. Doxycycline is an effective antimalarial drug. Although the mechanism of action of doxycycline against malaria is not fully understood, it is hypothesized that it may interfere with the synthesis of mitochondrial proteins. $^{10}$

Doxycycline monotherapy has been shown to be effective in cases of cutaneous leishmaniasis (CL). Masmoudi et al. ${ }^{11}$ prospectively treated 14 patients with CL with 15-30 days of doxycycline $200 \mathrm{mg}$ daily and demonstrated "complete desinfiltration" of the lesions in 10 patients (71\%). Malik et al. ${ }^{12}$ compared oral doxycycline $200 \mathrm{mg}$ daily to oral chloroquine $250 \mathrm{mg}$ twice daily in 50 patients with CL and found that 23 of 25 patients (92\%) who took doxycycline (for up to 12 weeks) had either complete re-epithelialization or healing of the lesions without induration.

A hamster experimental model of $\mathrm{VL}$ demonstrated a reduction in mortality at 3-5 weeks from $15 \%$ to $0 \%$ using doxycycline treatment, although the mechanism of action was unclear. ${ }^{13}$ Published human data of doxycycline use in VL are limited to a single case report. An immunocompetent Kosovan patient with a fever and dry cough was treated with 3 weeks of doxycycline $100 \mathrm{mg}$ twice daily because of positive leptospirosis serology, with complete resolution of symptoms. His symptoms recurred a month later, he was treated with a further 16 days of doxycycline, and they were attenuated but did not resolve. Amastigotes were found in the bone marrow, and serology confirmed a diagnosis of $L$. infantum. ${ }^{3}$

Our case and other published cases highlight a potential role for doxycycline as adjunctive treatment for VL. Liposomal amphotericin $B$ is the gold standard treatment for treating $V L$, but its use in endemic areas is limited by its price, as well as logistic issues relating to its storage (below $25^{\circ} \mathrm{C} / 77^{\circ} \mathrm{F}$ ) and administration (requires filtration, dilution, and slow intravenous infusion), ${ }^{14}$ and therefore exploration of alternative oral options is warranted. Given the relative low cost of doxycycline and its good safety profile, even when taken long term, it may be an appealing option, particularly in lower resource settings. The role of doxycycline as an adjunctive agent for treating VL merits further study.

Received October 2, 2019. Accepted for publication June 19, 2020.

Published online September 21, 2020.

Authors' addresses: Ankush Dhariwal, St Bartholomew's Hospital, Barts Health NHS Trust, London, United Kingdom, E-mail: ankush. dhariwal@nhs.net. Sarah Jawad and Tihana Bicanic, St George's University Hospitals NHS Foundation Trust, London, United Kingdom, E-mails: sarah.jawad@nhs.net and tihana.bicanic@nhs.net.

\section{REFERENCES}

1. Rahimian J, Raoult D, Tang YW, Hanna BA, 2006. Bartonella quintana endocarditis with positive serology for Coxiella burnetii. J Infect 53: e151-e153.

2. Edouard S, Million M, Casalta JP, Collart F, Amphoux B, Raoult D, 2017. Low antibodies titer and serological cross-reaction between Coxiella burnetii and Legionella pneumophila challenge the diagnosis of mediastinitis, an emerging $Q$ fever clinical entity. Infection 45: 911-915.

3. Heer K, Pfister D, Briner V, 1999. Revival of tetracyclines--in the treatment of visceral leishmaniasis? Schweiz Med Wochenschr 129: 810-812.

4. Caballero ZC, Sousa OE, Marques WP, Saez-Alquezar A, Umezawa ES, 2007. Evaluation of serological tests to identify Trypanosoma cruzi infection in humans and determine crossreactivity with Trypanosoma rangeli and Leishmania spp. Clin Vaccine Immunol 14: 1045-1049.

5. World Health Organization, 2019. Leishmaniasis Country Profiles-Spain. Geneva, Switzerland: WHO. Available at: https://www.who.int/leishmaniasis/burden/Leishmaniasis_ Spain/en/. Accessed December 17, 2019.

6. Centers for Disease Control and Prevention, 2017. Leishmaniasis Parasite Biology. Available at: https://www.cdc.gov/dpdx/ leishmaniasis/index.html. Accessed December 17, 2019.

7. Zijlstra EE, Siddig Ali M, El-Hassan AM, El-Toum IA, Satti M, Ghalib HW, Kager PA, 1992. Kala-azar: a comparative study of parasitological methods and the direct agglutination test in diagnosis. Trans R Soc Trop Med Hyg 86: 505-507. 
8. Piarroux R, Gambarelli F, Dumon H, Fontes M, Dunan S, Mary C, Toga B, Quilici M, 1994. Comparison of PCR with direct examination of bone marrow aspiration, myeloculture, and serology for diagnosis of visceral leishmaniasis in immunocompromised patients. J Clin Microbiol 32: 746-749.

9. da Silva MR, Stewart JM, Costa $\mathrm{CH}, 2005$. Sensitivity of bone marrow aspirates in the diagnosis of visceral leishmaniasis. $A m$ J Trop Med Hyg 72: 811-814.

10. Gaillard T, Madamet M, Pradines B, 2015. Tetracyclines in malaria. Malar J 14: 455.

11. Masmoudi A, Dammak A, Chaaben H, Maalej N, Akrout F, Turki H, 2008. Doxycycline for the treatment of cutaneous leishmaniasis. Dermatol Online J 14: 22.
12. Malik F, Hanif MM, Mustafa G, 2019. Comparing the efficacy of oral chloroquine versus oral tetracycline in the treatment of cutaneous leishmaniasis. J Coll Physicians Surg Pak 29: 403-405.

13. Alving CR, Steck EA, Chapman WL, Jr., Waits VB, Hendricks LD, Swartz GM, Jr., Hanson WL, 1980. Liposomes in leishmaniasis: therapeutic effects of antimonial drugs, 8-aminoquinolines, and tetracycline. Life Sci 26: 2231-2238.

14. Salih NA et al., 2014. Liposomal amphotericin B for complicated visceral leishmaniasis (kala-azar) in eastern Sudan: how effective is treatment for this neglected disease? Trop Med Int Health 19: $146-152$ 